\title{
La fidélisation : de la personnalisation à la tribalisation
}

\section{Bernard Cova}

\section{(2) OpenEdition}

1 Journals

Édition électronique

URL : http://journals.openedition.org/communicationorganisation/3236

DOI : 10.4000/communicationorganisation.3236

ISSN : $1775-3546$

Éditeur

Presses universitaires de Bordeaux

Édition imprimée

Date de publication : 1 juin 2005

Pagination : $32-43$

ISSN : $1168-5549$

\section{Référence électronique}

Bernard Cova, "La fidélisation : de la personnalisation à la tribalisation», Communication et

organisation [En ligne], 27 | 2005, mis en ligne le 07 juin 2012, consulté le 19 avril 2019. URL : http:// journals.openedition.org/communicationorganisation/3236; DOI : 10.4000/

communicationorganisation.3236

Ce document a été généré automatiquement le 19 avril 2019.

(c) Presses universitaires de Bordeaux 


\title{
La fidélisation : de la personnalisation à la tribalisation
}

\author{
Bernard Cova
}

1 Par fidélisation, on entend un programme d'actions organisées de telle manière que les clients les plus intéressants et les plus fidèles, soient stimulés et entretenus. Pour qu'un programme de fidélisation résiste aux attaques de la concurrence, il est nécessaire de construire et renforcer le lien entre l'entreprise et le client. Dans ce cadre, la vente d'un produit ou d'un service est considérée comme un épisode dans la relation entre l'entreprise ou/et la marque et le client. C'est la relation client qui est centrale à la stratégie marketing de l'entreprise, non la vente à ce client. Il s'agit alors d'optimiser la gestion de la relation client, c'est-à-dire de gérer tous les épisodes (commerciaux ou non) d'interaction entre le client et l'entreprise. Pour ce faire, les entreprises ont mis en place des démarches reposant sur la construction, le développement et le maintien d'un lien personnalisé avec le client.

2 Cette personnalisation des échanges au travers d'outils allant (Lehu, 1999) de la carte de fidélité au consumer magazine est le plus souvent supportée par un système informatique de traitement de l'information client connu sous le nom de CRM. Ce type de système a connu une énorme expansion au début des années 2000. Pourtant, il montre aujourd'hui des signes d'essoufflement qui amènent à reconsidérer de nombreuses hypothèses soutenant l'idée même de fidélisation par personnalisation des échanges. Les changements sociétaux définis comme postmodernes ainsi que les stratégies alternatives des entreprises et des marques en pointe conduisent à s'intéresser aujourd'hui aux pratiques de fidélisation reposant non sur un lien personnalisé mais sur un lien communautaire, dites de fidélisation par tribalisation. C'est ce que nous allons développer ici en essayant de mettre en lumière les avantages et les questions apportées par ce type de fidélisation. 


\section{La résistible ascension du CRM}

3 Le discours dominant en marketing aujourd'hui énonce que dans les marchés toujours plus concurrentiels auxquels elles doivent faire face, les entreprises ont intérêt à se construire une base de clients fidèles pour assurer leur fond de commerce. C'est le cas par exemple pour les hôtels, les restaurants, les chambres d'hôtes, les compagnies aériennes, etc. Une base de clients fidèles c'est tout à la fois un taux assuré d'occupation des équipements, la certitude d'un chiffre d'affaires minimum, une garantie de proximité et de convivialité avec une partie de la clientèle et un apport d'informations intimes pour faire évoluer l'offre. Il est donc normal que les entreprises aient cherché durant la dernière décennie à compléter la panoplie classique des outils marketing centrée sur la conquête de clients et de parts de marché, par des outils originaux centrés sur le développement et le maintien de relations de long terme avec un client fidélisé (Lehu, 1999). Au-delà des approches intuitives que tout un chacun a pu mettre en place, ces approches relationnelles, que l'on regroupe sous le vocable de marketing relationnel, se sont largement inspirées de ce que font les entreprises en milieu industriel depuis des décennies. En effet, dans un contexte industriel, que ce soit celui de l'aéronautique, de l'électrotechnique ou encore de l'énergie, les marchés sont semblables à des réseaux de relations avec peu de fournisseurs (une dizaine) et peu de clients significatifs (quelques dizaines tout au plus) qui maintiennent entre eux des relations de fidélité dépassant souvent les dix ans. Le marketing industriel consiste ainsi depuis toujours à créer, développer et maintenir des relations de long terme mutuellement profitables entre clients et fournisseurs (Michel et al., 2000).

Profitant des moyens nouveaux fournis par l'évolution technologique (base de données, Internet et autres), les entreprises de grande consommation venant de secteurs aussi divers que le tourisme, l'habillement ou encore l'automobile, ont donc cherché à développer des démarches dites de marketing relationnel (Hetzel, 2004) en s'inspirant des approches relationnelles existantes en milieu industriel. La grosse différence est que dans le cas des entreprises vendant des produits grand public, il s'agit de fidéliser des centaines de milliers de clients et non seulement quelques dizaines comme fait précédemment par les industriels! Fidéliser un client dans l'esprit du marketing de grande consommation, cela consiste de plus en plus à prendre un individu au berceau et à l'accompagner jusqu'à la vieillesse, en lui proposant, pour chacun des moments de sa vie et selon les événements qui les conditionnent, les produits et les services qu'il est susceptible d'utiliser et d'apprécier le plus. Cette personnalisation de l'offre et ce traitement sur mesure de la communication s'appuient, de la part de l'entreprise, sur une connaissance supposée intime de son client, de son environnement et de ses goûts. Le rapport ainsi établi avec un client minutieusement différencié et sélectionné pour sa valeur stratégique représente le capital le plus précieux de l'entreprise. L'accent mis sur l'importance de la gestion de la relation entre un client et l'entreprise est un credo si largement partagé aujourd'hui par un ensemble d'entreprises que beaucoup considèrent le CRM (Customer Relationship Management, ou GRC en français, Gestion de la Relation Client) comme un passage obligatoire.

5 Pourtant, ces approches montrent des signes évidents de limites dans leurs tentatives de fidélisation du client. Le Gartner Group parle même d'une désillusion pour parler de la période actuelle qui fait suite à la phase d'engouement du début des années 2000. En effet, 
55 \% des projets de CRM ne produisent pas les résultats escomptés selon le Gartner Group et le CRM est classé antépénultième dans un classement des 25 techniques managériales donnant le plus de satisfaction aux entreprises. Pire, le groupe Bain a mené une étude (Rigby et al., 2002) sur 451 cadres dirigeants qui montre qu'un cinquième d'entre eux considère que leur CRM n'a non seulement pas donné les résultats escomptés mais qu'en plus il a détruit certaine de leurs meilleures relations client de long terme! Comment peut-on expliquer une telle situation? Tout d'abord, il faut se rappeler que la performance d'un CRM dépend plus de la stratégie que du montant de l'investissement technologique! La stratégie cela veut dire allouer des ressources rares pour créer un avantage concurrentiel et ainsi délivrer une performance supérieure. Tout cela permettant au personnel de bien comprendre ce qu'il doit faire et ne pas faire au quotidien avec un client donné et c'est cela la clé de la qualité de l'interaction entreprise/ client. La technologie n'est qu'un investissement, la plupart du temps nécessaire (et encore, pas dans tous les secteurs), mais non suffisant et cet investissement doit s'accompagner d'un changement important du système des gestion du personnel. Beaucoup d'entreprises ont malheureusement fait le contraire : elles ont implanté une démarche de CRM avant d'avoir développé une véritable stratégie client et/ou elles ont mis en branle cette démarche avant d'avoir transformé l'organisation en conséquence. Ensuite, il faut noter que le CRM donne l'impression d'être contre-productif par rapport aux attentes latentes des clients (Fournier et al., 1998). En effet, il conduit parfois à des résultats contraires à ceux attendus: sentiment de violation de la vie privée, de déséquilibre dans la relation, de manipulation au travers de certains privilèges accordés, sans parler du renforcement du sentiment de déshumanisation de la relation et, en conséquence, d'isolement des individus clients. D'ailleurs, l'effort relationnel accompli par beaucoup d'entreprises en grande consommation n'est pas une réponse à une demande de relation de la part du consommateur, c'est bien plus l'effet de nouvelles techniques de gestion des contacts avec la clientèle. Cette exigence de relations ne résulte donc pas d'une nouvelle demande d'un soit disant nouveau consommateur; les études montrent, en effet, que peu de gens sont réellement prêts à payer plus pour avoir des produits et services personnalisés, contrairement à ce que proclament les tenants du marketing personnalisé. C'est bien plutôt l'intensification concurrentielle et la prolifération des instruments de communication mis à disposition des individus et des entreprises qui créent cette recherche de relation. Il est marquant que la plupart des relations initiées par le CRM restent des relations foncièrement déséquilibrées: l'entreprise peut contacter le client quand elle veut et, de plus en plus, où elle veut quand le client, lui, trouve souvent porte close ou n'arrive pas à joindre la personne qu'il désire et est renvoyé sur un call center! Aujourd'hui, beaucoup pensent qu'il faut dépasser la seule recherche de fidélisation du client pour développer une compréhension fine du contexte social dans lequel le client met en jeu les produits et les services offerts par l'entreprise. Comprendre le consommateur veut dire comprendre son vécu quotidien et pour cela il faut dépasser la base de données et aller sur le terrain pour «vivre avec les indigènes consommateurs » (Fournier et al., 1998).

\section{Le nécessaire passage à la communauté}

6 Cet intérêt pour tout ce qui touche au vécu quotidien du consommateur est sans doute un des changements les plus importants survenus en marketing ces dernières années : tout 
s'est passé comme si soudain, on réalisait l'importance de comprendre la dimension émotionnelle ou expérientielle de la consommation (Hetzel, 2002). Cette analyse du vécu quotidien du consommateur met en évidence que le consommateur actuel, loin de poursuivre une course effrénée vers la seule personnalisation, recherche paradoxalement à retrouver du lien social et de la communauté au sein de regroupements à saveur tribale (Maffesoli, 1988). Toutes les passions ordinaires partagées avec d'autres individus passionnés sont ainsi le signe d'individus en recherche, plus ou moins réelle ou virtuelle, de communauté émotionnelle. En conséquence, le marketing devrait donc moins chercher à faire un lien personnalisé avec un client qu'à soutenir le lien entre clients en les aidant à partager leurs passions. Il y a maintenant une décennie que le marketing tribal a ouvert la porte à des approches dites communautaires de la consommation (Cova, 1995). Le comportement de consommateurs réunis en groupe par une même passion ou un même ethos, développé dans une sous culture ou tribu, a alors commencé à être considéré comme un objet d'étude pertinent pour le marketing. En parallèle, l'avènement d'Internet a amené l'idée que les marques pouvaient créer des communautés de consommateurs autour de leurs sites Web. Réintégrant les récents travaux sur la relation complexe entre les marques et les consommateurs à cette idée de communauté de consommateurs, le concept de communauté de marque (brand community) est alors apparu sur le devant de la scène marketing où il voisine avec celui de tribu de marque( brand tribe). Le magazine Business Week a ainsi consacré son tout dernier classement (9 Août 2004) des 100 premières marques mondiales à la montée des communautés de marque et à leur rôle très positif pour la santé des marques concernées considérées alors comme des marques tribales (Apple avec son I-Pod, Harley-Davidson, Starbucks et autres). Ce faisant, une partie de la fidélisation évolue vers ce que certains nomment la fidélisation tribale. Aujourd'hui de nombreux travaux de recherche en marketing essaient ainsi d'évaluer les effets du sentiment d'appartenance à une communauté de marque sur la fidélité du consommateur à la marque (McAlexander et al., 2002 et 2003 ; Rosenbaum et al., 2004).

7 Rappelons qu'en marketing (Lehu, 1999), on oppose la fidélité comportementale dans laquelle un consommateur est considéré fidèle lorsqu'il achète régulièrement la même marque à la fidélité attitudinale dans laquelle un consommateur est considéré fidèle à la marque lorsqu'il l'achète régulièrement et qu'il a développé à son égard une attitude favorable. Dans cette fidélité attitudinale, il faut différencier encore entre fidélité cognitive et fidélité affective. La fidélité cognitive se définit comme l'attachement rationnel du consommateur à la marque ; la fidélité affective est plutôt un attachement émotionnel fondé sur une relation durable caractérisée par la dépendance du consommateur vis-à-vis de la marque et la présence de sentiments durables d'amitié pour la marque. Selon les derniers travaux de recherche cités précédemment, il apparaît que la fidélisation par tribalisation permet un développement beaucoup plus important de la fidélité affective que ne le permet la fidélisation par personnalisation qui développe plutôt la fidélité cognitive. Et dans une société postmoderne où la consommation prend une place de plus en plus importante dans la définition identitaire de l'individu (Cova et Cova, 2001) au travers de la communion et de l'émotion générée par une marque ou un produit, la fidélité affective devient un objectif majeur des stratégies marketing (Oliver, 1999). La force du lien pluriel ainsi créé entre consommateurs et supporté par la marque ou l'entreprise est bien plus forte que la force du lien dyadique entre l'entreprise et le consommateur: on ne casse pas facilement un lien affectif avec un groupe de gens avec 
lesquels on a conscience de former un groupe à part et pour lesquels on a une obligation morale d'entraide (Muniz et O'Guinn, 2001). Pour ces consommateurs, le lien entre les membres devient souvent plus important que le bien (Cova, 1995), c'est-à-dire le produit support de la passion partagée. De plus, l'existence de rituels et de traditions aide et oblige le consommateur à perpétuer le lien. C'est ce qu'ont bien compris les entreprises produisant des motos comme Ducati et Harley-Davidson qui multiplient les évènements rituels (des randonnées notamment) locaux, nationaux et internationaux ou celles produisant des chaussures comme Nike ou Adidas qui créent de véritables temples à leur effigie dans lesquels se regroupent les fidèles (c'est le cas de le dire !).

8 Mais, si la fidélisation par tribalisation, c'est-à-dire au travers de la mise en jeu d'une tribu de marque, semble présenter des avantages très nets par rapport à la fidélisation par personnalisation, comme décrit dans le tableau 1, il n'apparait par contre pas du tout facile de savoir comment l'entreprise peut communiquer avec ce collectif de consommateurs pour développer la fidélité de ses membres. Les concepts de rituels, de lieux de culte, de brandfests ou fêtes de la marque (McAlexander et Schouten, 1998) sont encore loin d'être des lieux communs de la pratique marketing.

Tableau 1 : Comparaison des Pratiques de Fidélisation

\begin{tabular}{|l|l|l|l|}
\hline & $\begin{array}{l}\text { FIDELISATION } \\
\text { PERSONNALISATION }\end{array}$ & $\begin{array}{l}\text { FIDELISATION } \\
\text { TRIBALISATION }\end{array}$ \\
\hline Relation de Référence & Client - Entreprise & Client - Client - Client... Entreprise \\
\hline $\begin{array}{l}\text { Position } \\
\text { l'Entreprise }\end{array}$ & $\begin{array}{l}\text { L'entreprise comme pôle de la } \\
\text { relation }\end{array}$ & $\begin{array}{l}\text { L'entreprise comme support des } \\
\text { relations }\end{array}$ \\
\hline $\begin{array}{l}\text { Moyens } \\
\text { Fidélisation }\end{array}$ & $\begin{array}{l}\text { Carte de fidélité, lettre } \\
\text { d'informations... }\end{array}$ & Rituels, lieux de culte, brandfests... \\
\hline $\begin{array}{l}\text { Type Dominant de } \\
\text { Fidélité }\end{array}$ & Fidélité cognitive & Fidélité affective \\
\hline
\end{tabular}

Pour communiquer avec une tribu de marque, l'entreprise doit donc changer de registre mais aussi changer de grandeur: il faut passer du discours marchand au discours non marchand. Par exemple, l'entreprise Salomon qui communique beaucoup avec des tribus de glisse évite soigneusement de faire toute fausse note qui pourrait la faire accuser d'être une vulgaire world company. Dans ses actions terrain, Salomon bannit tout ce qui ne concerne pas la passion de la tribu. L'enjeu d'un concours local pour des snowboarders ne sera pas un téléphone portable, mais le droit de faire une démonstration devant les grands champions. Salomon crée ses propres opérations (plutôt que de se greffer sur des opérations existantes) qui obéissent à la même logique : faire avancer la pratique tribale en mettant en place de grands rendez-vous pour les passionnés. Elle a le souci de ne pas faire de récupération, mais bien plutôt de bannir toute forme de sponsoring superficiel. Salomon veille aussi à ce que toute sa communication soit la moins agressive possible. Salomon cherche moins à mettre en avant son image qu'à s'inscrire comme membre à part entière des différents mouvements tribaux au même plan qu'un passionné individuel. Enfin, communiquer avec une ou des tribus de marque à des fins de 
fidélisation, comme nous venons de l'imager par l'exemple de Salomon, implique une perte de contrôle, ou au moins de volonté de contrôle, de l'entreprise dans sa relation avec son marché et ses clients. Il ne s'agit pas de contrôler les tribus en communiquant unilatéralement vers ses tribus mais bien d'interagir avec elles en reconnaissant leurs compétences et leurs expertises.

\section{Lâcher du lest dans la communication}

En effet, dans la vision managériale dominante des relations producteur/consommateur, l'entreprise sait produire ce qui est bon pour le consommateur car elle en a l'expertise et le consommateur est considéré comme un naïf. Aujourd'hui, dans de nombreux domaines (bricolage, informatique, santé) cette césure n'existe plus, l'expertise est autant chez le consommateur que chez le producteur. Il y a montée des compétences du consommateur et construction collective de connaissances, toutes choses renvoyant à l'idée d' empowerment des consommateurs. Les Anglo-saxons (Keat et al., 1994) ont nommé cela, il y a déjà longtemps, "l'autorité des consommateurs ». Rares sont les entreprises capables de reconnaître cette expertise; on peut citer Wizards, distributeur des jeux de rôle Dungeons and Dragons, qui rémunère (royalties) les joueurs ayant apporté des améliorations notables à un jeu, ou, dans le même secteur, Ubi Soft, distributeur du jeu Morrowind, qui met en ligne des patchs informatiques corrigeant certains défauts du jeu et réalisés par des joueurs-experts. Pour la petite histoire Ubi Soft a reconnu que ces patches, réalisés par des joueurs, étaient plus performants que celui réalisé en interne par ses ingénieurs.

11 Ceci est encore plus vrai en ce qui concerne les tribus de marque au-dedans desquelles les passionnés échangent et enrichissent mutuellement leurs compétences. Ces tribus de passionnés construisent ainsi en leur sein, par les échanges fréquents et fortement chargés émotionnellement, une expertise que l'entreprise serait bienvenue de reconnaître et de mettre en jeu (Cova et Carrère, 2002). Les tribus de passionnés représentent des communautés d'experts prêts à s'investir dans la vie de leur marque chérie. Ces tribus sont moins des communautés d'intérêt cherchant à se regrouper pour négocier, que des communautés de passion où se partage un discours sans retenue ni tabou sur l'ensemble de la marque : ses valeurs, ses produits passés, présents et à venir, les points de vente, le design, la publicité, la technique, les expériences vécues de chacun ou encore les ressentis. Ainsi, dans les tribus de passionnés d'une marque automobile, chaque annonce de nouveau modèle ou nouveau projet, chaque interview du dirigeant, chaque nouvelle publicité, est analysée, disséquée, discutée, mise en perspective. Si l'entreprise sait communiquer avec elles de manière vraiment interactive en reconnaissant leur expertise, ces tribus sont alors de formidables supports de démultiplication des programmes de fidélisation.

Le besoin d'interaction et de dialogue est encore plus accentué quand on interagit avec la tribu de marque via le Net (Kozinets, 1999 et 2002). De récentes expériences ont mis en lumière toute la difficulté qu'il pouvait y avoir pour l'entreprise à interagir avec ce type d'acteur collectif difficilement contrôlable que le Net fait spontanément émerger et grossir (O'Guinn et Muniz, 2004). La question récurrente en terme de fidélisation est de savoir quel niveau de contrôle l'entreprise peut avoir sur le site et les échanges de sa tribu de marque. Agir avec cet acteur collectif ne se réduit pas à identifier et suivre un ou 
des leaders d'opinion, mais bien à collaborer avec un ensemble humain, détenteur et générateur de compétences collectives.

Pour essayer de trouver des modes opératoires sur le Net entre communautés de consommateurs et entreprises, on a fait un parallèle avec les travaux menés (Callon et al., 2001) sur la coopération entre chercheurs et communautés de malades ou de riverains de sites pollués pour faire avancer à la fois la science et le bien-être quotidien au travers de compromis socio-techniques. Il en est ressorti la proposition de développer des sites sous la forme de «forums hybrides» (Callon et al., 2001). On parle de forums, parce qu'il s'agit d'espaces ouverts où des groupes peuvent se mobiliser pour débattre de choix qui engagent le collectif. Et l'on ajoute le qualificatif hybrides, parce que ces groupes engagés et les porte-parole qui prétendent les représenter sont hétérogènes : on y trouve à la fois des experts, des hommes politiques, des techniciens et des profanes qui s'estiment concernés. Ces forums sont qualifiés d'hybrides, également, parce que les questions abordées et les problèmes soulevés s'inscrivent dans des registres variés qui vont de l'éthique à l'économie en passant par la physiologie, la sociologie et même le marketing. Réutilisé dans le contexte de la fidélisation par tribalisation, le concept de forum hybride pourrait se traduire par la création d'un site officiel et collectif qui accepte une grande diversité d'opinions et s'ouvre à la coopération, au degré décidé par l'entreprise (design des produits, design du site, adaptation de l'offre), avec la tribu de marque. L'entreprise montrerait ainsi qu'elle a identifié et reconnu l'expertise de ses passionnés. Dès lors, il lui serait possible de justifier sa position, de dialoguer avec les groupes de passionnés, d'expliquer ce qu'elle met en place pour s'améliorer en s'appuyant sur les contributions des diverses tribus de consommateurs. La récente tentative faite par le groupe Ferrero avec le site my nutella The Community est intéressante à cet égard.

\section{Le cas my nutella The Community}

Partant du principe que Nutella est un produit convivial par excellence, le grand groupe agro-alimentaire transalpin Ferrero a tout récemment décidé de favoriser l'émergence d'une communauté, au niveau italien pour l'instant, de passionnés de Nutella. D'où l'idée du site my nutella The Community (www.mynutella.com) qui marie la Nutella avec Internet qui est la technologie par excellence par laquelle se manifeste la spontanéité sociale et la mise en réseau des individus. Selon Antonio Massimo Castiglia, Responsable Internet et Multimédia de Ferrero, ce site est un projet de communication ambitieux qui part d'un renversement complet de la relation entre la marque et les consommateurs. Au travers de my nutella The Community, la marque fait « un pas en arrière " comme il le dit car elle laisse la scène aux consommateurs qui deviennent les protagonistes et les artisans de la relation. Le site my nutella The Community se veut l'agora à laquelle adhèrent et participent tous ceux qui s'identifient avec les valeurs du produit, valeurs qui vont bien au-delà des caractéristiques uniques et particulières du produit et touchent à de nombreux aspects de la vie sociale. Le site est un chantier ouvert qui évolue et prend forme en fonction de ce qu'en font les participants. Le site my nutella The Community regroupe (mi-2004) un réseau de 10000 internautes italiens passionnés de Nutella. Sur ce site, chaque passionné peut créer son propre site en commençant par écrire son Nutella Pensiero, un court texte sur Nutella, relatant notamment sa première expérience, sa vision de la marque ou tout autre pensée sur la Nutella. Sur ce site personnel, il peut aussi publier un journal racontant sa vie avec Nutella (ou non) et détailler ses hobbies. Les autres membres peuvent 
commenter publiquement (guestbooks) ou en privé (emails). De plus, chacun peut mettre en ligne ses photos les plus réussies dans la section Nutella Live notamment celles prises au cours d'une des fameuses Nutella Parties qui sont des rituels communautaires offline mettant en jeu la Nutella. Une autre section du site est destinée aux fans clubs Nutella. Certains passionnés s'y regroupent pour former un fan club online sans forcément qu'il y ait une correspondance offline. La plupart de ces fans clubs sont, en effet, des agrégations virtuelles, sortes de sous-groupes au sein de la tribu de marque. Cette section contient aussi l'annuaire de tous les fans club Nutella d'Italie. Il y a encore la section Nutella Art\&Collection avec un nombre significatif de pots de collection et la section MyBacheca (Mon panneau d'affichage) qui est le guide officiel de tous les produits dérivés autour de la Nutella (gadgets, livres, films et autres).

Sur le site my nutella The Community, les passionnés de Nutella ont donc à disposition de nombreuses options pour communiquer avec d'autres consommateurs envahis par la même passion. La volonté affichée du groupe Ferrero est, avec ce site, de transformer le potentiel de valeur de lien de son produit en créant un réseau communautaire de participants dans lequel se développera la fidélisation par tribalisation; l'ensemble devant se rapprocher d'un forum hybride. Pour ce faire, Ferrero a organisé le site my nutella The Community de façon à faciliter "l'exposition de soi» (Cauquelin, 2003) des consommateurs et, par là même, le marketing personnel de chaque consommateur et de chaque groupe de consommateurs, et non celui du producteur. Ferrero s'est appuyé sur le besoin existentiel de reconnaissance qui passe par la perception : «j'existe si je suis perçu. Essere est percepi, suivant la fameuse formule du philosophe immatérialiste anglais, évêque de surcroît, Georges Berkeley» (Cauquelin, 2003). Aujourd'hui, le consommateur recherche, en effet, des possibilités d'anonymat intime ou d'intimité exposée : une intimité déballée sur des sites qui ne sont pas ouverts à tous les vents mais seulement à ceux qui présentent les mêmes symptômes passionnels que le consommateur concerné, c'est-à-dire les membres de la tribu. Et c'est bien ce que propose Ferrero avec le site my nutella The Community qui est une structure d'accueil formelle pour tous ceux qui ont besoin de se faire entendre et se sentir exister au travers de la Nutella. Seule différence importante par rapport à la notion de forum hybride : il n'y a pas, pour l'instant, d'ouverture à la coopération de la part de Ferrero dans la définition des produits Nutella.

Compte tenu du changement sociétal actuel, un programme de fidélisation ne peut plus rimer seulement avec personnalisation. Il doit aussi intégrer la fidélisation par tribalisation, c'est-à-dire la fidélisation par inclusion du consommateur dans une communauté ou tribu de marque. Cette fidélisation beaucoup plus affective peut porter à de très hauts degrés de fidélité, proche de la foi religieuse, mais requiert une approche de communication de l'entreprise foncièrement en rupture avec ce qu'elle a l'habitude de faire : il ne s'agit plus de contrôler les messages envoyés vers les consommateurs mais plutôt d'aider ces derniers à s'exprimer soit offline soit online en reconnaissant par là même les compétences et l'expertise qu'ils ont pu développer seuls et en groupes à partir de l'expérience vécue avec la marque et ses produits. 


\section{BIBLIOGRAPHIE}

Callon, M., Lascoumes, P., Barthe, Y., Agir dans un monde incertain : essai sur la démocratie technique, Paris, Seuil, 2001.

Cauquelin, A., L'exposition de soi : du journal intime aux Webcams, Paris, Eshel, 2003.

Cova, B., Au-delà du marché : quand le lien importe plus que le bien, Paris, L'Harmattan, 1995.

Cova, B. et Carrère, V., « Les communautés de passionnés de marque : opportunité ou menace sur le Net », in : Revue Française du Marketing, № 189/190, 2002, pp. 119-130.

Cova, V. et Cova, B., Alternatives Marketing, Paris, Dunod, 2001.

Fournier, S., Dobscha, S. et Mick, D.G., « Preventing the Premature Death of Relationship

Marketing ", in: Harvard Business Review, Jan-Feb, 1998, pp. 42-51.

Hetzel, P., Planète conso : marketing expérientiel et nouveaux univers de consommation, Paris, Editions d'Organisation, 2002.

Hetzel, P., Le marketing relationnel, Paris, Puf, 2004.

Keat, R., Whiteley, N. et Abercrombie, N., The Authority of the Consumer, Londres, Routledge, 1994. Kozinets, R.V., « E-Tribalized Marketing? The Strategic Implications of Virtual Communities of Consumption », in: European Management Journal, vol. 17, N 3, 1999, pp. 252-264.

Kozinets, R.V., « The Field Behind the Screen: Using Netnography for Marketing Research in Online Communities », in: Journal of Marketing Research, vol. XXXIX, February, 2002, pp. 61-72. Lehu, J.M., La fidélisation client, Paris, Editions d'Organisation, 1999.

Maffesoli, M., Le temps des tribus. Le déclin de l'individualisme dans les sociétés postmodernes, Paris, Méridiens, 1988 et LVP ( 2 ème édition), 2000.

McAlexander, J.H., Kim, S.K. et Roberts, S.C., « Loyalty: The Influences of Satisfaction and Brand Community Integration », in: Journal of Marketing Theory \& Practice, vol. 11, Nº 4, 2003, pp. 1-17.

McAlexander, J.H. et Schouten, J.W., « Brandfests: Servicescapes for the Cultivation of Brand Equity », in Sherry, J.F. (ed.), Servicescapes: The Concept of Place in Contemporary Markets, Lincolnwood, IL, NTC Business Books, 1998, pp. 377- 401.

McAlexander, J.H., Schouten, J.W. et Koenig, H.F., « Building Brand Community », in: Journal of Marketing, vol. 66, January, 2002, pp. 38-54.

Michel, D., Salle, R. et Valla, J.P., Marketing industriel : Stratégies et mise en œuvre, 2 ème édition, Paris, Economica, 2000.

Muniz, A. M. Jr. et O'Guinn, T.C., « Brand Community », in: Journal of Consumer Research, vol. 27, March, 2001, pp. 412-432.

Oliver, R., « Whence Customer Loyalty? », in: Journal of Marketing, vol. 63, January, 1999, pp. 33-44.

Rigby, D.K., Reicheld, F.K. et Scheffer, P., « Avoid the Four Perils of CRM », in: Harvard Business Review, Feb., 2002, pp. 101-109. 
Rosenbaum, M., Ostrom, A.L. et Kuntze, R., « Loyalty Programs and A Sense of Community », in: Journal of Services Marketing, vol. 18, 2004.

\section{RÉSUMÉS}

Alors que l'approche classique de la fidélité tend à créer et développer un lien entre la marque et le client, l'approche dite tribale de la fidélité préfère (re)créer et soutenir le lien entre clients. Les interrelations communautaires exercent, en effet, une pression sur le client pour qu'il reste fidèle au groupe et par là même à la marque. Dans cette perspective, l'ensemble des moyens de communication est dédié à soutenir ce lien communautaire et non à le remplacer.

Whereas the traditional approach to loyalty aims at creating and developing a relation between the brand and a customer, the tribal approach to loyalty prefers to recreate and support the relation between customers. The communal inter-relations exert pressure on members to remain loyal to the collective and consequently to the brand. In this view, all the means of communication are dedicated to supporting the tribal link, not substituting for it.

\section{AUTEUR}

\section{BERNARD COVA}

Professeur ESCP-EAP Paris et Responsable du Laboratoire Savoir Sud/Euromed Marseille. Son domaine de recherche est le marketing dans une perspective postmoderne. Fondés sur un point de vue ethnosociologique, ses travaux sur les approches sociétales de la consommation ont dès 1995 posé les bases des développements actuels sur le rôle du marketing et du design dans la reconstruction de liens sociaux entre individus. Mail : bcova@escp-eap.net 